\title{
Prevalence and characteristics of persistent symptoms after non-severe COVID-19: a prospective cohort study
}

\author{
Lucas Armange $^{1} \cdot$ François Bénézit ${ }^{1} \cdot$ Léa Picard ${ }^{1} \cdot$ Charlotte Pronier $^{2} \cdot$ Stéphanie Guillot $^{3} \cdot$ Pierre-Axel Lentz $^{4}$. \\ François Carré ${ }^{5}$. Pierre Tattevin ${ }^{1}$ (1) $\cdot$ Matthieu Revest ${ }^{1}$
}

Received: 8 February 2021 / Accepted: 19 April 2021 / Published online: 23 April 2021

(C) The Author(s), under exclusive licence to Springer-Verlag GmbH Germany, part of Springer Nature 2021

\begin{abstract}
We performed a prospective cohort study of 311 outpatients with non-severe COVID-19 (187 women, median age 39 years). Of the $214(68.8 \%)$ who completed the 6-week follow-up questionnaire, $115(53.7 \%)$ had recovered. Others mostly reported dyspnea $(n=86,40.2 \%)$, weight loss $(n=83,38.8 \%)$, sleep disorders $(n=68,31.8 \%)$, and anxiety $(n=56,26.2 \%)$. Of those who developed ageusia and anosmia, these symptoms were still present at week 6 in, respectively, 11/111 (9.9\%) and 19/114 (16.7\%). Chest CT scan and lung function tests found no explanation in the most disabled patients $(n=23)$. This study confirms the high prevalence of persistent symptoms after non-severe COVID-19.
\end{abstract}

Keywords COVID-19 $\cdot$ Persistent symptoms $\cdot$ Prospective cohort $\cdot$ Dyspnea $\cdot$ Investigations $\cdot$ Asthenia

\section{Introduction}

With $>50$ million cases reported in 2020, the acute phase of coronavirus disease 2019 (COVID-19) has been well characterized, especially in patients who required hospital admission [1], but few studies focused on persistent symptoms [2-4]. The characteristics and outcome of COVID-19 in patients who were not admitted have attracted less attention [5]. However, physicians have been confronted with patients complaining of persistent symptoms after non-severe COVID-19 [6]. We aimed to assess the prevalence of symptoms 6 weeks after non-severe COVID-19 in a prospective cohort of outpatients.

Pierre Tattevin and Matthieu Revest contributed equally to this work.

Pierre Tattevin

pierre.tattevin@chu-rennes.fr

1 Infectious Diseases and Intensive Care Unit, Pontchaillou University Hospital, Rue Henri Le Guilloux, 35033 Rennes, France

2 Virology, Pontchaillou University Hospital, Rennes, France

3 Respiratory Physiology, Pontchaillou University Hospital, Rennes, France

4 Radiology, Pontchaillou University Hospital, Rennes, France

5 Cardiology and Sport Medicine, Pontchaillou University Hospital, Rennes, France

\section{Methods}

We conducted a monocentric observational study at the Rennes University Hospital, a tertiary-care hospital that serves as a referral center for COVID-19 in western France (population catchment area, 1.8 million inhabitants), from March 3rd to June 23th, 2020. We enrolled all adults outpatients diagnosed with COVID-19 in the infectious diseases ambulatory unit, who presented symptoms suggestive of COVID-19 associated with either a positive SARS-CoV-2 RT-PCR from a nasopharyngeal swab and/or close contact with biologically proven case(s) and who consented to participate in the study. All patients were followed through a digital application on smartphone, with daily questionnaires about symptoms, including fever, respiratory, and digestive signs, pain, mood, and weight loss during the first 3 weeks. At any moment, they could ask questions through the application to doctors or trained medical students or request for consultation. First day of follow-up was defined as the day of COVID-19 diagnosis.

At 6 weeks of follow-up, a link to an anonymized webbased questionnaire was sent to all patients enrolled in the prospective cohort, by e-mail. This short questionnaire collected data on respiratory symptoms, smell and taste disorders, general status, and mood (Supplementary table). Patients were offered to be contacted by one investigator (LA). For patients who were unable to resume their pre-COVID-19 activities, a consultation was planned within 2 weeks, for comprehensive 
medical evaluation, including anamnesis and physical examination, to determine whether additional investigations and/or treatment were necessary.

When indicated, investigations were performed within 1 month, and included non-contrast chest CT scan, and lung function tests (LFT). Patients who primarily complained of asthenia and motor weakness were managed in an outpatient rehabilitation center (sports medicine department). Patients with persistent smell and/or taste disorders were offered to participate in a specific rehabilitation program for anosmia/ ageusia. Patients with disabling persistent respiratory symptoms were evaluated by a pulmonologist, who could initiate treatment based on LFT findings (e.g., short or long-acting bronchodilators and/or inhaled corticosteroids). Quantitative data are reported as median [interquartile range] and qualitative data as number (\%). This study was approved by the Rennes University Hospital institutional review board.

\section{Results}

We enrolled 311 patients, with a median age of 39 years [2951], and a female-to-male ratio of 187/124 (1.5). For 216 patients $(69.5 \%)$, COVID-19 was documented by positive PCR on nasopharyngeal samples. The remaining presented flu-like illness of acute onset following close contact with a PCR-positive case, and/or associated with anosmia/ageusia, and or with suggestive thoracic CT scan. Among the 311 patients enrolled, 214 (68.8\%) completed the web-based questionnaire at 6 weeks (Fig. 1). Major findings at 6-week follow- up were as follows: $115 / 214$ patients $(53.7 \%)$ had fully recovered. Others mostly reported dyspnea $(n=86,40.2 \%)$, weight loss $(n=83,38.8 \%$, with a median weight loss of $2 \mathrm{~kg}[1-3])$, sleep disorders $(n=68,31.8 \%)$, anxiety $(n=56,26.2 \%)$, cough $(n=41,19.2 \%)$, sadness $(27,12.6 \%)$, and chest pain $(n=23,10.7 \%)$. Patients reported unusual dyspnea when going to the bathroom $(6 / 200,3.0 \%)$, taking a shower (16/ $203,7.9 \%)$, climbing stairs $(63 / 210,30.0 \%)$, or when practicing sports $(76 / 206,36.9 \%)$. Among the 151 patients who regularly practiced sports before their COVID-19, 84 (55.6\%) had resumed their sport activities. Among patients who suffered from smell or taste disorders during the acute phase of COVID-19, these symptoms were still present at week 6 in 11/ $111(9.9 \%)$ for ageusia and 19/114 (16.7\%) for anosmia, with a median time-to-recovery at 2 weeks (Table 1 ).

The 23 patients who were most disabled by respiratory symptoms underwent LFT and chest CT scan, despite their physical examination was unremarkable. They were mostly women $(18 / 23,78.3 \%)$, with a median age of 44 years (3450). Comorbidities included asthma $(n=4)$ and smoking $(n=$ 2). Their COVID-19 had been documented by PCR in 13/23 $(56.5 \%)$, and their persistent symptoms included dyspnea $(n=$ $19,82.6 \%)$, asthenia $(n=9,39.1 \%)$, thoracic pain $(n=6$, $26.1 \%)$, and cough $(n=4,17.4 \%)$. LFT documented decreased diffusing capacity of the lung for carbon monoxide (DLCO) in 9 patients (39.1\%), mild-to-moderate in all (mean, $64.5 \%$ normal value, minimum 56\%), including the 2 patients with active smoking. One patient had mild restrictive syndrome. Two patients presented mild desaturation during the 6-min walk test, but never $<95 \% \mathrm{SaO}_{2}$. The median distance

Fig. 1 Study flow chart

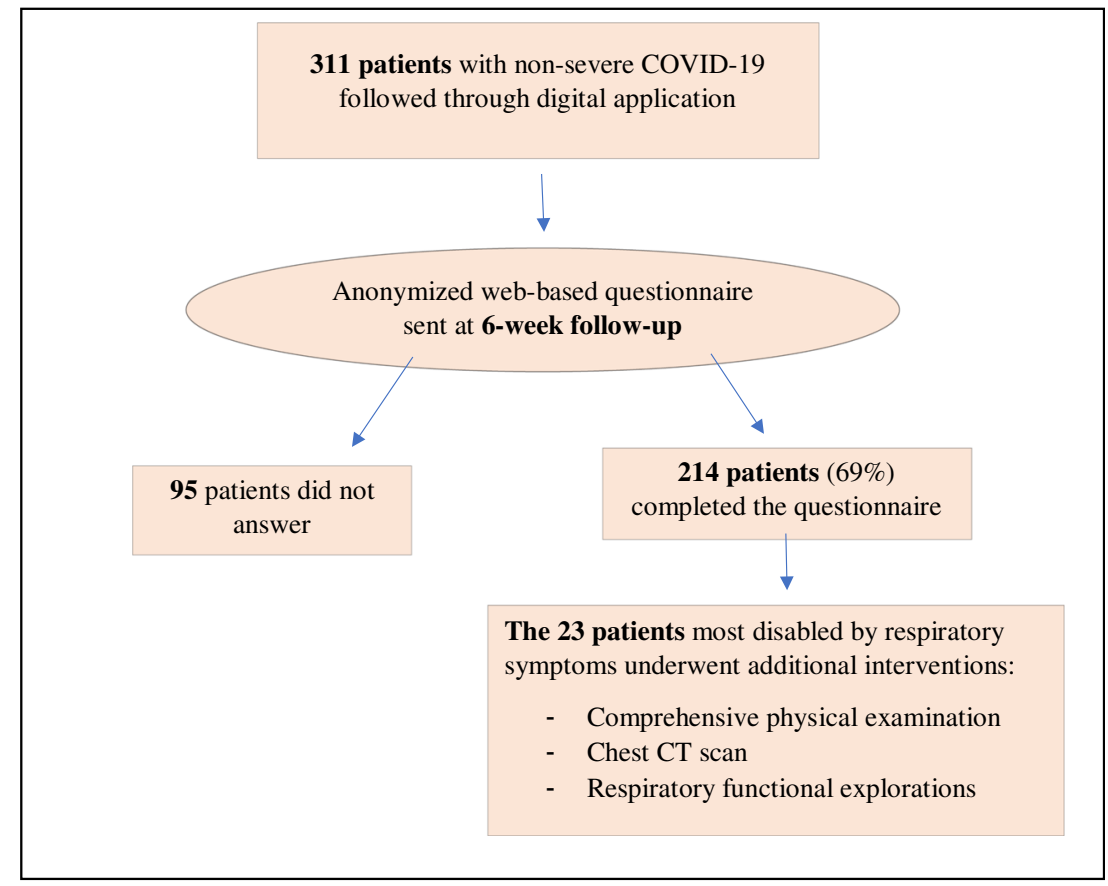


Table 1 Non-severe COVID-19 patients baseline characteristics and 6week follow-up

\begin{tabular}{lc} 
Baseline $(n=311)$ & \\
\hline Age, years & $39[29-51]$ \\
\hline Female sex & $187(60.1 \%)$ \\
At least one comorbidity & $61(19.6 \%)$ \\
Active smoking & $25(8.0 \%)$ \\
Chronic respiratory disease & $25(8.0 \%)$ \\
Chronic heart disease & $14(4.5 \%)$ \\
Six-week health status $(n=214)$ & \\
Returned to baseline health status, no residual symptom & $115(53.7 \%)$ \\
Dyspnea & $86(40.2 \%)$ \\
- Only on exertion & $76(35.6 \%)$ \\
- Permanent & $10(4.7 \%)$ \\
Cough & $41(19.2 \%)$ \\
Chest pain & $23(10.7 \%)$ \\
Weight loss & $83(38.8 \%)$ \\
Sleep disorders & $68(31.8 \%)$ \\
Anxiety & $56(26.2 \%)$ \\
Sadness & $27(12.6 \%)$ \\
Respiratory functional explorations $(n=23)$ & \\
Decreased DLCO & $9 / 23(39 \%)$ \\
Clinical bronchial hyper-reactivity & $7 / 23(30 \%)$ \\
Restrictive syndrome & $1 / 23(4 \%)$ \\
Obstructive syndrome & $0 / 23(0 \%)$ \\
Chest CT scan findings $(n=23)$ & \\
Ground glass lesions* & $4(17 \%)$ \\
Pulmonary fibrosis & 0 \\
\hline & \\
\hline & \\
\hline
\end{tabular}

Quantitative data are reported as median [interquartile range] and qualitative data as number (\%)

$D L C O$ diffusing capacity of the lung for carbon monoxide

*In all cases (4/4), lesions were significantly improved as compared to baseline CT scan

covered during this exercise was 90\% (64-95) of theoretical values. The median Borg rating of perceived exertion scale was 5/10 [3-5]. Clinical bronchial hyper-reactivity was detected in 7 patients $(30.4 \%)$, and hyperventilation syndrome suspected in three (13.0\%). Following LFT, a treatment by bronchodilators and/or inhaled corticosteroids was introduced in 11 patients $(47.8 \%)$.

Chest CT scan did not find any pattern of pulmonary fibrosis. Ground-glass opacities were detected in 4 patients $(17.4 \%)$, significantly improved as compared with initial chest CT scan in all (Fig. 2). Of note, the 4 patients with persistent CT scan abnormalities had decreased DLCO. Fifteen patients were transferred to the sports medicine rehabilitation center, and six initiated rehabilitation program for anosmia/ageusia.

\section{Discussion}

The major findings of this prospective cohort study are the followings: (i) even in patients with non-severe COVID-19, total resolution of symptoms takes time: approximately $50 \%$ of patients had not returned to their baseline health status 6 weeks after their COVID-19, and only $55 \%$ of those who practiced sports before were able to resume their sport activities; (ii) the primary complaints, at 6 weeks, included dyspnea, weight loss, sleep disorders, and anxiety; and (iii) even among patients most affected by post-COVID symptoms, physical examination was unremarkable, while LFT and chest CT scan were normal in most cases.

Little is known about the persistence of disabling symptoms following COVID-19. A post-acute outpatient service for individuals discharged from hospital after recovery from COVID-19 in Italy found that only 18/143 patients (12.6\%) were completely free of COVID-19related symptoms at 60 days. Fatigue, dyspnea, pain, and lack of appetite were reported by $>50 \%$ of patients [2]. Similar findings were reported from Ireland, where $52.3 \%$ of patients $(67 / 128)$ reported persistent fatigue 10 weeks after COVID-19, and this was not associated with severity during the acute COVID-19 [3]. A multicenter study from Egypt found higher prevalence of fatigue in patients who recovered from COVID-19 (209/287, $72.8 \%$ ) [4]. To our knowledge, our study is the first to provide systematic investigations in patients most disabled by respiratory symptoms: All of them (23/23) had normal physical examination, no new findings on chest CT-scan, and no or limited abnormalities on LFT.

The optimal management of patients with persistent symptoms after the acute phase of COVID-19 remains unknown. A Post-COVID-19 Functional Status (PCFS) scale has been proposed to evaluate the entire range of functional limitations, including in lifestyle, sports, and social activities [7]. Others advocated for an interdisciplinary approach, through a post-COVID outpatient unit, to implement comprehensive and individualized care for these patients $[6,8,9]$.

Our study has limitations. First, our web-based questionnaire was deliberately short, to ensure a high proportion of answers: hence, it was not powered to detect all postCOVID symptoms. Second, we only collected data at 6 weeks, so that we have no information beyond this time point. Additional studies are requested to estimate symptoms duration, as this is one of the main concerns for patients. Third, investigations were restricted to patients most disabled and were limited to non-contrast chest CT scan and LFT. However, in our experience, other investigations, including contrast-enhanced CT scan, echocardiography, and tests for inflammatory or auto-immune disorders, provide no 
Fig. 2 Non-contrast chest CT scan. a, b Consolidations and ground-glass opacities with crazy paving at baseline in 2 patients. $\mathbf{c}$, d Same patients, control at 10 weeks with nearly complete resolution

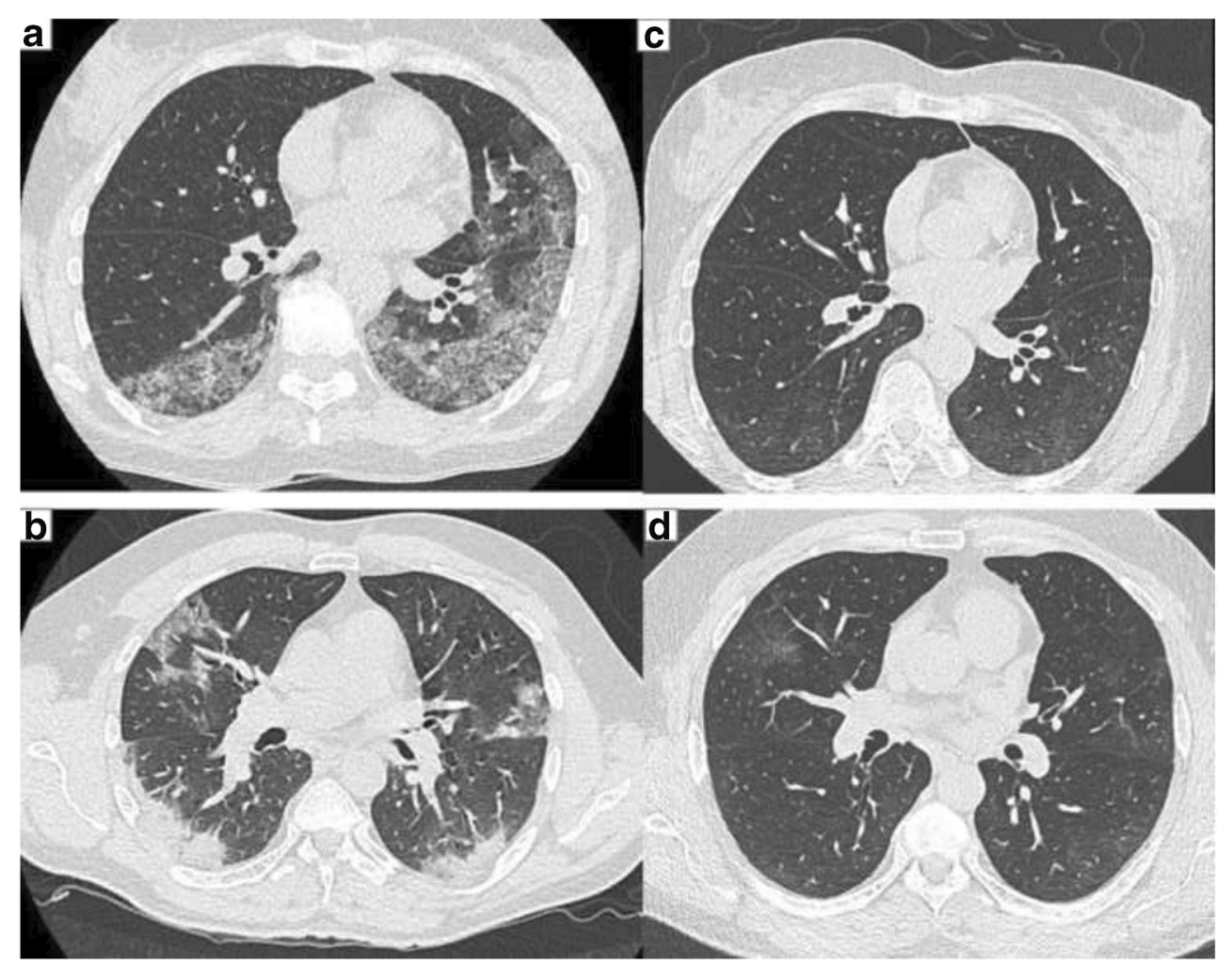

additional information about the pathophysiology of persistent symptoms. Fourth, our study population was young (median age, 39 years), with a female predominance. This was related to inclusion criteria (non-severe COVID cases), and to the fact that, due to shortages, tests were restricted to inpatients and health care workers, in France, during the study period. Hence, our findings may not apply to other population.

In conclusion, this prospective cohort study confirms the high prevalence of persistent symptoms even after non-severe COVID-19. Long-term follow-up with multidisciplinary management is requested to assist patients throughout this difficult recovery time.

Supplementary Information The online version contains supplementary material available at https://doi.org/10.1007/s10096-021-04261-y.

\section{Code availability Not applicable.}

Author contribution LA, FB, MR, and PT participated in study design, collected data, and wrote the first draft of the manuscript. All authors were involved in patients care, revised, and approved the final version of the manuscript.

Data availability On request.

\section{Declarations}

Ethics approval The study was approved by the Ethic Committee of Rennes University Hospital.
Consent to participate Waived.

Consent for publication Waived.

Competing interests The authors declare no competing interests.

\section{References}

1. Zhou F, Yu T, Du R, Fan G, Liu Y, Liu Z et al (2020 Mar) Clinical course and risk factors for mortality of adult inpatients with COVID19 in Wuhan, China: a retrospective cohort study. Lancet 395(10229):1054-1062

2. Carfì A, Bernabei R, Landi F, for the Gemelli against COVID-19 post-acute care study group. Persistent Symptoms in Patients After Acute COVID-19. JAMA [Internet]. 2020 Jul 9 [cited 2020 Jul 13]; Available from: https://jamanetwork.com/journals/jama/fullarticle/ 2768351

3. Townsend L, Dyer AH, Jones K, Dunne J, Mooney A, Gaffney F et al (2020) Persistent fatigue following SARS-CoV-2 infection is common and independent of severity of initial infection. PLoS One 15(11):e0240784

4. Kamal M, Abo Omirah M, Hussein A, Saeed H (2020 Sep) Assessment and characterisation of post-COVID-19 manifestations. Int J Clin Pract 29:e13746

5. Lutchmansingh DD, Knauert MP, Antin-Ozerkis DE, Chupp G, Cohn L, Dela Cruz CS et al (2020 Nov) A clinic blueprint for postCOVID-19 RECOVERY: learning from the past, looking to the future. Chest. 4

6. Landi F, Gremese E, Bernabei R, Fantoni M, Gasbarrini A, Settanni CR et al (2020 Aug 1) Post-COVID-19 global health strategies: the need for an interdisciplinary approach. Aging Clin Exp Res 32(8): $1613-1620$ 
7. Klok FA, Boon GJAM, Barco S, Endres M, Geelhoed JJM, Knauss S, et al. (2020) The Post-COVID-19 Functional Status scale: a tool to measure functional status over time after COVID-19. Eur Respir J. 56(1).

8. Barker-Davies RM, O'Sullivan O, Senaratne KPP, Baker P, Cranley M, Dharm-Datta S et al (2020 Aug) The Stanford Hall consensus statement for post-COVID-19 rehabilitation. Br J Sports Med 54(16):949-959
9. Davido B, Seang S, Tubiana R, de Truchis P (2020) Post-COVID-19 chronic symptoms: a postinfectious entity? Clin Microbiol Infect

Publisher's note Springer Nature remains neutral with regard to jurisdictional claims in published maps and institutional affiliations. 